\title{
Milking it for all it's worth: The effects of environmental enrichment on maternal nurturance, lactation quality, and offspring social behavior
}

Holly DeRosa ${ }^{1}$, Hieu Tran ${ }^{1}$, Amanda C. Kentner ${ }^{1 *}$

School of Arts \& Sciences, Health Psychology Program, Massachusetts College of Pharmacy and Health Sciences, Boston Massachusetts, United States 02115

*Corresponding author:

Amanda Kentner

amanda.kentner@mcphs.edu

Office \#617-274-3360

Fax \# 617-732-2959

Keywords

Environmental enrichment; lactation; nutrition; social behavior; prenatal experience; postnatal experience; press posture; microbiome; milk; breastfeeding 


\begin{abstract}
While alterations in the neonatal environment can have dramatic effects on offspring outcomes, the mechanisms that drive this phenomenon remain unclear. Breastfeeding confers robust benefits to offspring development, including those related to growth, immunity, and neurophysiology. Similarly, improving environmental complexity (i.e., environmental enrichment; EE) contributes developmental advantages to both humans and laboratory animal models. However, the impact of environmental context on maternal care and milk quality has not been thoroughly evaluated, nor are the biological underpinnings of EE on offspring development understood. Here, Sprague-Dawley rats were housed and bred in either EE or standard-housed (SD) conditions. Although EE dams gave birth to a larger number of pups, litters were standardized and cross-fostered across groups on postnatal day (P)1. Maternal milk samples were then collected on P1 (transitional milk phase) and P10 (mature milk phase) for analysis. While EE dams spent less time nursing, postnatal enrichment exposure was associated with heavier offspring bodyweights. Moreover, milk from EE dams had increased triglyceride levels compared to SD dams. Milk from EE mothers also contained a greater microbiome diversity and a significantly higher abundance of bacterial families related to bodyweight and energy metabolism. In addition to changes in lactational quality, we observed sex- and time-dependent effects of $\mathrm{EE}$ on offspring social behavior. Specifically, prenatal EE exposure was associated with greater sociability in females, while postnatal EE was associated with greater sociability in male offspring. Together, these results underscore the multidimensional impact of the combined neonatal and maternal environments on offspring development. These data also identify potential deficiencies in the quality of the "gold standard" laboratory housing condition and its impact on the welfare and design of translationally relevant animal models in biomedical research.
\end{abstract}

\title{
1. Introduction
}

The maternal-infant interaction of breastfeeding is a critical component in the development of offspring. The nutritional value of breastmilk is largely recognized, as it contains a cocktail of fats, proteins, carbohydrates, vitamins, enzymes, and immunoglobulins that infants need for healthy development. (Boix-Amorós et al., 2019; Lessen \& Kavanagh, 2015). Milk microbes can directly influence the development of the infant intestinal tract and gut microbiome (Sheard \& Walker, 1988; Pannaraj et al., 2017). Breastfeeding during infancy also confers offspring immunity against certain illnesses, including type 1 diabetes, gastrointestinal diseases, and even COVID-19 (Frank et al., 2019; Hanson, 1998;

Sadauskaitė $\square$ Kuehne et al., 2004; Gray et al., 2021; Romero Ramírez et al., 2021).

Unlike commercial formula, breastmilk naturally contains nutritional elements such as betaine and the omega-3 fatty acid docosahexaenoic acid (DHA), which are directly involved in offspring metabolism and brain health (Ribo et al., 2021; Lauritzen et al., 2016; Andreas et al., 2014). Breastmilk also contributes to infant neural development by promoting, increased white and grey matter volume, improved cortical thickness (Isaacs et al., 2008; Luby et al., 2016) and neuromuscular development (Grace et al., 2017). Some studies have even linked performance on certain intelligence parameters to breastfeeding (Horwood \& Fergusson, 1998; Kanazawa, 2015; Luby et al., 2016) although socioeconomic factors should be considered (Brion et al., 2011). Milk may facilitate development of the central nervous system by directly influencing genes related to neural growth and maturation. For example, microRNAs in mouse milk were found to target proteins in the offspring small intestine related to neuron proliferation, differentiation, and survival (Huff et al., 2020). Together, this 
evidence implicates milk in brain development, although the role of milk in offspring behavior needs further elucidation.

The profile of breastmilk can be modulated by the maternal environment, and several studies have revealed the pervasive effects of stress on milk production and quality. For example, mothers who have been exposed to natural disasters often report a reduction or sometimes complete loss of lactation (Adhisivam et al., 2006; DeYoung et al., 2018). However, displacement by such extreme environmental occurrences can be associated with severe maternal malnourishment, which similarly hinders milk production. This is in addition to reducing milk vitamin and mineral content (Smit et al., 2000; Spring et al., 1985;

Sauerwald et al., 2001). However, even non-disastrous contexts such as psychosocial stress negatively correlates with maternal milk fat and energy content (Ziomkiewicz et al., 2021) and mothers who reported higher levels of perceived stress exhibited significantly lower levels of milk immunoglobulin A (Moirasgenti et al., 2019; Groer et al., 2004). Stress arising from perinatal anxiety and depression can also be associated with significant differences in milk composition, including a reduction in DHA, increased sodium, and increased transforming growth factor $\beta-2$ (reviewed in Di Benedetto et al., 2021). Likewise, changes in the environment correlate with altered maternal milk quality in laboratory animals. For example, nursing mice fed a high-fat diet demonstrated an increase in milk lactose and fat content as well as differently expressed mRNAs related to central nervous system development (Chen et al., 2017). Restraint stress has been shown to reduce milk protein in lactating mice (Chiba et al., 2019) and other stressors such as social and heat stress have been shown to negatively affect lactation (Murgatroyd et al., 2015) and milk yield (Haldar \& Bade, 1981) respectively. Such evidence underscores how the maternal environment may contribute to alterations in the nutritional profile of milk which may have subsequent consequences on progeny development, although effects on offspring remain largely unexplored.

The enhancement of environmental complexity (i.e., environmental enrichment or $\mathrm{EE}$ ) is employed in healthy human populations to promote cognitive plasticity (Baroncelli et al., 2010; Kentner et al., 2019a; Kentner et al., 2019b; Tooley et al., 2021). Additionally, EE housing reduces stress and stereotypy and promotes species typical behaviors in the animal laboratory. This enhanced housing condition has been shown to affect the display of rodent maternal care behaviors (Welberg et al., 2006; Connors et al., 2015; Cancedda et al., 2004; Mann \& Gervais, 2011; Strzelewicz et al., 2019; Sale et al., 2004). Quality of maternal behavior during the lactational period is central to the development of effective stress regulation and health of the offspring (Meaney \& Szyf, 2005; Meaney, 2010; Buschdorf \& Meaney, 2011). Research has supported the notion that EE dams are more efficient mothers compared to their standard-housed (SD) counterparts. For example, while rats housed in EE spent less time on their nest compared to SD dams, both groups licked and groomed their pups at a similar (Welberg et al., 2006; Connors et al., 2015; Strzelewicz et al., 2019) or even higher (Sale et al., 2004) frequency, although these findings are not always consistent (Cancedda et al., 2004; Rosenfeld \& Weller, 2012). Additionally, EE dams have been shown to spend less time in a passive nursing posture compared to SD dams and demonstrate higher levels of the more effective active, or high arched back nursing posture (Connors et al., 2015; Strzelewicz et al., 2019). In another study, rat dams housed in cages with a loft that afforded an opportunity to periodically leave their pups also exhibited lower levels of passive nursing (Ratuski \& Weary, 2021). This suggests that time away from the pups promotes maternal care efficiency upon returning to the nest. This is congruent with what is typically observed under naturalistic conditions, where wild rat dams will leave their nests for extended periods to forage and defend their territory (Hughes et al., 1978; Grota \& Ader, 1969).

Given that the SD dams housed in the classic "gold standard" laboratory housing condition spend more time on the nest suggests these animals may overfeed their offspring. 
Alternatively, there may be metabolic differences in milk quality between the housing conditions where the SD offspring require more nourishment, necessitating longer nursing periods. Indeed, dams in cages with reduced opportunities to leave their litters spend more time in a "press posture" position with the ventral surface of their body pressed against the cage, hiding their teats from their pups (Gaskill \& Pritchett-Corning, 2015; Cramer et al., 1990). In the long term, this has implications for laboratory animal health and stress regulation. Therefore, in the current study we explored the effects of EE versus SD housing on rodent maternal care, maternal milk quality, and offspring body weight and social behavior outcomes in male and female rats.

\section{Materials and Methods}

\subsection{Animals and housing}

Sprague-Dawley rats (Charles River, Wilmington, MA) were maintained at $20^{\circ} \mathrm{C}$ on a 12 h light/dark cycle (0700-1900 light) with ad libitum access to food and water. Figure 1A outlines the experimental procedures followed in this study. Female animals were pairhoused in one of two conditions: environmental enrichment $(\mathrm{EE} ; 91.5 \times 64 \times 159 \mathrm{~cm}$; see Figure 1B), which was a large multi- level cage with ramps and access to toys, tubes, chew bones, and Nestlets ${ }^{\circledR}$ (Ancare, Bellmore, NY), or standard laboratory cages (SD; $27 \times 48 \times 20$ $\mathrm{cm}$; see Figure 1C). Enrichment toys (e.g., small plastic balls, climbing ropes and ladders, swings, bell rollers, chew toys, hammocks, additional tubes/tunnels, Lixit Space Pods, cups, and other small animal hideaways) were switched out twice weekly to maintain novelty in the EE condition.

Male rats were paired in SD conditions unless they were breeding. During breeding, they were housed with two females in either EE housing or in larger SD one-level cages $(51 \times 41 \times 22 \mathrm{~cm})$ with access to a tube, one chew bone and Nestlets ${ }^{\circledR}$ (Ancare, Bellmore, NY). Approximately two days prior to parturition, dams in the SD condition were individually housed $(27 \times 48 \times 20 \mathrm{~cm}$; Figure 1C), while a physical divider separated the EE dams within their cage (allowing for auditory, tactile, olfactory, and some visual contact; important components of EE). This separation prevented the mixing of litters. Day of birth was designated as postnatal day (P) 0 and litters were standardized to 10 pups per litter on P1. To dissociate the effects of the pre- and postnatal housing environments, male and female pups from each litter were cross-fostered at this time. To track the housing of origin, pups were marked on their left or right ear to indicate prenatal SD or EE respectively, resulting in the following study group designations: SD-SD, SD-EE, EE-SD, EE-EE. Offspring were maintained in these respective housing conditions until the end of the study on P22. Animal procedures were conducted in accordance with the Association for Assessment and Accreditation of Laboratory Animal Care with protocols approved by the Massachusetts College of Pharmacy and Health Sciences (MCPHS) Institutional Animal Care and Use Committee.

\subsection{Milk sample collection}

To encourage the accumulation of maternal milk for sample collection, on the mornings of P1 (equivalent to a transitional milk phase) and P10 (mature milk phase), dams were removed from their litter and placed into a clean cage in a separate procedure room for one hour. Pups remained in their regular holding room and were placed into a smaller clean cage positioned on top of a heating pad, to maintain their body temperature. Litters were weighed 
immediately prior to being returned to their dams and again 2 and 24 hours later, alongside the inspection of milk bands, to monitor their health.

The milk procedure was adapted from a published procedure (Paul et al., 2015). Immediately following the separation period, dams were lightly anesthetized with isoflurane in $\mathrm{O} 2$, followed by the administration of $0.2 \mathrm{~mL}$ of oxytocin (20 USP/mL i.p). Distilled water was used to moisten the teats and milk obtained by gently squeezing its base to manually expel the sample for collection. A microhematocrit tube was used to collect $\sim 20 \mathrm{ul}$ of sample. The tube was then sealed and placed into a hematocrit spinner and spun for $120 \mathrm{sec}$ at 13, 700 g. Measurements of the separation of the milk into cream and clear layers were taken to calculate percent $(\%)$ creamatocrit using the procedures outlined in Paul et al. (2015). The remaining milk collected ( $\sim 500 \mathrm{ul}$ per animal) was transferred to small centrifuge tubes and stored at -80 degrees Celsius until processing. Collection time took about 10-20 minutes per animal, and dams were returned to their litter as soon as they awoke from anesthesia. This was appropriate as breastfeeding can resume immediately after isoflurane anesthesia since the pharmacokinetics of the compound indicate it is poorly absorbed by infants (Drugs and Lactation Database, 2020; Lee \& Rubin, 1993). With respect to oxytocin, its typical half-life (1-6 minutes) is reduced even further during lactation and this drug is also unlikely to affect offspring (Par Pharmaceutical Inc, 2020).

\subsection{Milk sample analyses}

Milk samples $(n=8)$ were placed onto a Mini Tube Rotator (Fisher Scientific Cat. \#88861051) overnight at $4^{\circ} \mathrm{C}$ to homogenize prior to analysis. Following the standard manufacturer's instructions, commercially available ELISA kits were used to measure lactose content (Sigma-Aldrich Cat. \#MAK017; diluted 1:500; as outlined by Chen et al., 2017), triglycerides (Abcam, Cat. \#ab65336; 1:1000 dilution), protein (Pierce ${ }^{\mathrm{TM}}$ BCA Protein Assay Kit; Cat. \#23227; 1:50 dilution), and immunoglobulin (Ig) A (Bethyl Laboratories, Cat. \# E111-102; diluted to 1:1000) levels in milk. To measure corticosterone, the small sample assay protocol (\#ADI-900-097, Enzo Life Sciences, Farmingdale, NY) was followed, as recommended by the manufacturer, using a 1:40 dilution. We opted to only evaluate P10 milk samples on these measures because litters were appropriately standardized in size for this time point.

Another subset of P10 milk samples underwent microbiome community analysis $(n=6)$. Milk DNA was first extracted using the ZymoBIOMICS ${ }^{\circledR}-96$ MagBead DNA Kit (Zymo Research, Irvine, CA). The Quick-16S ${ }^{\mathrm{TM}}$ NGS Library Prep Kit (Zymo Research, Irvine, CA) was used for bacterial 16S ribosomal RNA targeted sequencing and custom 16S primers were utilized to amplify the V3-V4 region (Zymo Research, Irvine, CA). Real-time PCR was then used to prepare the sequencing library and final qPCR fluorescence readings were pooled together according to equal molarity and the final pooled library was cleaned using the Select-a-Size DNA Clean \& Concentrator ${ }^{\text {TM }}$ (Zymo Research, Irvine, CA), and quantified with TapeStation ${ }^{\circledR}$ (Agilent Technologies, Santa Clara, CA) and Qubit ${ }^{\circledR}$ (Thermo Fisher Scientific, Waltham, WA). Illumina ${ }^{\circledR}$ MiSeq $^{\mathrm{TM}}$ with a v3 reagent kit (600 cycles) was used along with a $10 \%$ PhiX spike-in to sequence the final library. Unique amplicon sequences, as well as possible sequencing errors and chimeric sequences, were inferred from raw reads using the DADA2 pipeline (Callahan et al., 2016). Uclust from Qiime (v.1.9.1) was used to determine taxonomy assignment and referenced with the Zymo Research Database (Zymo Research, Irvine, CA).

\subsection{Maternal care}


Maternal behavior observations took place between P1-P4 and again on P10 following the milking procedures $(n=8)$. Sessions occurred three times daily $(7: 30,15: 00,20: 00)$, consisting of six observations resulting in a composite score for each dam and observation period. Dams were evaluated for 1-minute intervals per observation, with at least 5 minutes of no observations occurring between each of the 1-minute bins. Maternal care observations recorded included the frequency of pup-directed behaviors (i.e., dam licking/grooming pups, active/high crouch nursing, passive/low crouch nursing, pup retrieval), self-directed behaviors (i.e., dam eating/drinking, dam self-grooming, dam chasing her arm/tail), and nest building/digging behavior. Total time the dam spent on her nest (seconds) was also recorded (Strzelewicz et al., 2021; Strzelewicz et al., 2019; Connors et al., 2015).

\subsection{Open field and social preference tests}

On P21, one male and one female offspring per litter from each pre- versus postnatal housing condition was habituated to an open field arena for three-minutes $(40 \mathrm{~cm} \times 40 \mathrm{~cm} \times$ $28 \mathrm{~cm}$; Duque-Wilckens et al., 2020; Williams et al., 2020, $\mathrm{n}=7-8$ ). Behavior was recorded and videos scored using an automated behavioral monitoring software program (Cleversys TopScan, Reston, VA) to determine total distance traveled $(\mathrm{cm})$ and percent of time spent in the center of the arena. All equipment was thoroughly cleaned with Quatriside TB between each animal and test. Immediately after the open field habituation period, animals were evaluated in a five-minute social preference test. Using the manual behavioral monitoring program ODLogTM 2.0 (http://www.macropodsoftware. com/), animals were evaluated on their choice to visit a novel inanimate object or a novel same sex and age conspecific, each enclosed within a small wire cup on opposite ends of the arena (Crawley, 2007). Placement of novel rats and objects were interchanged between trials and experimental groups counterbalanced between tests. Animals were recorded as actively investigating when their nose was directed within $2 \mathrm{~cm}$ of a containment cup, or it was touching the cup. Percent time in contact with either the novel rat or object was calculated by the formula ([total time with target cup (rat or object) / 300 seconds] *100). Latency (seconds) to approach the novel rat was also recorded (Strzelewicz et al., 2019).

In a separate group of rat dams $(n=9)$, a pup preference test was run using a similar protocol. The purpose of this test was to determine if dams had a social preference for either SD or EE housed P7 pups, which may have impacted maternal care and later offspring social behavior (Champagne et al., 2009; Cromwell, 2011). Dams were assessed on the amount of time they spent exploring alien SD versus alien EE housed pups. One male and one female pup from each housing condition were placed into wire cups situated on opposite sides of an open field arena. The total duration (seconds) that SD and EE dams spent with each housing group across a ten-minute period was reported. On the morning of P22, a mixture of ketamine/xylazine $(150 \mathrm{mg} / \mathrm{kg}$, i.p. $/ 50 \mathrm{mg} / \mathrm{kg}$, i.p.) was used to anesthetize dams and their litters

\subsection{Statistical analysis}

Statistics were performed using the software package Statistical Software for the Social Sciences (SPSS) version 26.0 (IBM, Armonk, NY) or GraphPad Prism (version 9.0). The dataset was not powered to evaluate sex-differences so male and female animals were evaluated separately (Ordoñes Sanchez et al., 2021). Two-way repeated measure ANOVAs (Housing x Time) were used to compare P1 and P10 milk levels of creamatocrit, which is linearly related to the fat concentration and energy content of milk (Lucas et al., 1978; Paul et 
al., 2015). This statistical analysis was also used to compare the total time the dam spent on the nest (P1-P4, P10) across the light and dark phases of the circadian cycle.

A paired samples t-test was used to evaluate maternal preferences for P7 SD and EE housed pups. One-way ANOVAs were used to evaluate other measures of maternal care and milk composition (e.g., ELISA data) as a function of housing condition. In rare cases of violations to the assumption of normality (Shapiro-Wilk test), Kruskal-Wallis tests were employed (expressed as $X^{2}$ ). Offspring behavior was assessed using 2 x 2 (prenatal treatment $\mathrm{x}$ postnatal treatment) ANOVAs and LSD post hocs were applied except where there were fewer than three levels, in which case pairwise t-tests and Levene's (applied in the occurrence of unequal variances) were utilized alongside Bonferroni alpha adjustments.

Data are graphically expressed as mean \pm SEM. The partial eta-squared $\left(n_{p}{ }^{2}\right)$ is also reported as an index of effect size for the ANOVAs (the range of values being $0.02=$ small effect, 0.13 = moderate effect, 0.26 = large effect; Miles and Shevlin, 2001).

For the microbiome analyses, composition visualization, alpha-diversity, and betadiversity analyses were performed with Qiime (v.1.9.1) and statistical comparisons were performed using Kruskal-Wallis tests (Caporaso et al., 2010). To determine taxa that were significantly different between groups, linear discriminant analysis effect size (LEfSe; http://huttenhower.sph.harvard.edu/lefse/) was employed as previously described (Segata et al., 2011; Schellekens et al., 2021). In short, LEfSe creates a model that identifies taxa that are most likely to explain differences between groups through the use of a series of nonparametric tests (Segata et al., 2011).

\section{Results}

\subsection{Maternal housing condition affects maternal care}

One-Way ANOVA showed that EE dams gave birth to larger litters than their SD housed counterparts (SD: $14.0 \pm 0.60$ vs. EE: $16.63 \pm 1.05 ; \mathrm{F}(1,14)=4.173, \mathrm{p}=0.048, n_{p}{ }^{2}=0.252$; Figure 1D); all litters were standardized to 10 pups on P1. On P7, a social preference test showed that EE and SD dams investigated all pups to the same extent, regardless of the pups housing origin $(t(17)=0.808, p=0.430$; Figure 1E).

A repeated measures ANOVA suggested that the time dams spent on the nest did not change as a function of postnatal day across the light and dark periods ( $p>0.05$; Figure 1F, G). However, when these two observation periods were collapsed together a significant main effect of postnatal day emerged $\left(\mathrm{F}(4,56)=2.740, \mathrm{p}=0.037, n_{p}{ }^{2}=0.164\right.$; Figure 1H) with more time being spent on the nest on P3 (p = 0.013) and P4 ( $<<0.001)$, an effect driven by the SD group. Indeed, both the dark, and the total light + dark periods combined revealed a significant main effect of housing condition in that SD dams spent more time on the maternal nest than EE dams (dark: $\mathrm{F}(1,14)=16.987, \mathrm{p}=0.001, n_{p}{ }^{2}=0.548$; Figure 1G; total light + dark: $\mathrm{F}(1,14)=9.839, \mathrm{p}=0.007, n_{p}{ }^{2}=0.413$; Figure 1H). This general pattern persisted when the total time dams spent on the nest was summed into a composite score across the postnatal observation days. Again, SD dams were shown to spend more time on the nest than EE mothers (light: p>0.05, Figure 1I; dark: $X^{2}(1)=7.456, p=0.006$, Figure 1J; total light + dark: $X^{2}(1)=4.864, p=0.027$; Figure 1K). During our maternal care observations, we did not plan to quantify "press posture" (Gaskill \& Pritchett-Corning, 2015; Cramer et al., 1990; Ratuski \& Weary 2021) but we subjectively noted its presence in SD dams when it was noticed during our study. A representative photo of this posture can be found in Figure 1C. We did not observe this behavior in any EE dams. 
Maternal nursing postures did not differ as a function of housing in the light phase ( $p>0.05$; Figure 1L). However, the number of total nursing postures observed were significantly lower in EE dams during the dark phase (high arched back: $F(1,14)=24.462$, $p$ $=0.001, n_{p}^{2}=0.636$; passive/blanket: $X^{2}(1)=4.498, \mathrm{p}=0.034$; total nursing: $\mathrm{F}(1,14)=$ 17.996, $\mathrm{p}=0.001, n_{p}{ }^{2}=0.562$; Figure $\left.1 \mathrm{M}\right)$ and in the total combined light + dark phases (high arched back: $\mathrm{F}(1,14)=10.772, \mathrm{p}=0.005, n_{p}{ }^{2}=0.435$; passive/blanket: $\mathrm{F}(1,14)=$ 5.296, $\mathrm{p}=0.037, n_{p}{ }^{2}=0.274$; total nursing: $X^{2}(1)=9.289, \mathrm{p}=0.002$; Figure $1 \mathbf{N}$ ).

Maternal nest building bouts were significantly increased in SD dams during the light phase $\left(X^{2}(1)=7.323, p=0.007\right.$; Figure 10). SD dams also licked/groomed their pups more frequently in the dark phase $\left(\mathrm{F}(1,14)=22.257, \mathrm{p}=0.001, n_{p}{ }^{2}=.614\right.$; Figure 1P). However, the total amount of licking and grooming that $\mathrm{EE}$ and SD pups received across the total light + dark phases did not differ ( $p>0.05$; Figure 1Q), while increased nest building was sustained in SD dams $\left(X^{2}(1)=5.370, p=0.020\right.$; Figure 1Q).

During the light phase, maternal self-directed grooming and eating/drinking behaviors were elevated in SD dams (maternal self-grooming: $\mathrm{F}(1,14)=6.077, \mathrm{p}=0.027, n_{p}{ }^{2}=0.303$; eating/drinking: $\mathrm{F}(1,14)=4.833, \mathrm{p}=0.045, n_{p}{ }^{2}=0.257$; Figure 1R). SD dams also displayed a higher number of repetitive tail/arm chase behaviors (see Figure 1C for photograph) across the nychthemeron (light: $X^{2}(1)=6.536, p=0.011$; dark: $X^{2}(1)=6.303$, p $=0.012$, Figure 1S; total light + dark: $X^{2}(1)=6.792, p=0.009$; Figure 1T) and higher selfgrooming levels when the light + dark periods were combined $(F(1,14)=9.914, p=0.007$, $n_{p}{ }^{2}=0.415$; Figure 1T).

\subsection{Maternal housing condition affects lactation quality}

Given that SD dams spent significantly more time on the nest feeding their litters than EE dams, we evaluated whether there were differences in milk quality between the two housing conditions (see Figure 2A for photograph of milk collection procedure). There was a main effect of postnatal day for $\%$ creamatocrit, which is directly proportional to the fat concentration and energy content of milk (Lucas et al., 1978; Paul et al., 2015). These proportionally related measures decreased in both housing groups between $\mathrm{P} 1$ and $\mathrm{P} 10(\mathrm{~F}(1$, $14)=23.607, \mathrm{p}=0.001, n_{p}{ }^{2}=0.001$; Figure 2B, C, D) The ratio of different milk contents changes over the course of lactation in rats, and fat in particular decreases with time (Keen et al., 1981). There were no significant housing effects in the concentration of protein, lactose, corticosterone, or IgA in P10 milk ( $p>0.05$; Figure 2E, F, G, H). However, triglyceride levels were significantly higher in the milk of EE compared to SD housed dams $(\mathrm{F}(1,14)=$ 9.314, $\mathrm{p}=0.009, n_{p}{ }^{2}=0.400$; Figure 2I).

Housing condition also contributed to significant differences in the composition of the milk microbiome (Figure 2J, K). LEfSe analysis revealed 44 discriminative taxa between our housing groups, 38 of which were more highly expressed in the milk of EE dams.

Specifically, milk from EE dams demonstrated a significantly greater abundance of the phylum Tenericutes $($ LDA effect size $=3.39, \mathrm{p}=0.03$; Figure 2J). Additionally, Christensenellaceae $($ LDA effect size $=3.39 ; \mathrm{p}=0.007)$, Peptococcaceae $($ LDA effect size $=$ 3.499; $\mathrm{p}=0.02$ ), Coriobacteriaceae (LDA effect size $=3.59 ; \mathrm{p}=0.006)$, Lachnospiraceae $($ LDA effect size $=3.32 ; \mathrm{p}=0.007)$, Ruminococcaceae $($ LDA effect size $=4.73 ; \mathrm{p}=0.02)$ and Erysipelotrichaceae (LDA effect size $=3.33 ; \mathrm{p}=0.02$ ) were higher at the family level (Figure 2J, K) in EE mothers. Milk from SD dams had greater levels of Streptococcaceae (LDA effect size $=4.38 ; \mathrm{p}=0.02$; Figure 2J, K). Overall, EE milk contained greater species diversity compared to SD milk, as indicated by alpha diversity along the Shannon index $\left(\left(X^{2}(1)=5.77, p=0.016\right.\right.$; Figure 2L) and Bray-Curtis dissimilarity measurement of beta 
diversity $(\mathrm{R}=0.2944, \mathrm{p}=0.02$; Figure 2M). Please see Supplementary Figures 1 and 2 for the Cladogram of milk biomarkers and taxonomy heatmap respectively.

\subsection{Enrichment housing affects physiology and social behavior as a function of early life exposure}

A two-way (prenatal x postnatal) ANOVA revealed a main effect of postnatal experience for male and female offspring P21 body weights (males: $X^{2}(1)=9.562, p=0.002$; females: $X^{2}(1)=11.733, p=0.001$, Figure 3A, B, C). Postnatal enrichment housing resulted in significantly higher body weights than SD housing (males SD: $40.43 \pm 1.10 \mathrm{~g}$ versus EE: $50.86 \pm 2.01 \mathrm{~g}$; females SD: $40.191 \pm 0.99 \mathrm{~g}$ versus EE: $50.50 \pm 2.14 \mathrm{~g}$ ).

There was a main effect of postnatal experience for total distance traveled in that SD female offspring traveled more than EE females (SD: $262.98 \pm 56.69 \mathrm{~cm}$ vs EE: $130.41 \pm$ $22.76 \mathrm{~cm} ; X^{2}(1)=3.882 \mathrm{p}=0.049$; males: $\mathrm{p}>0.05$; Figure 3D, E, F). A main effect of prenatal experience was found for male animals $\left(X^{2}(1)=7.618, p=0.006\right.$; females: $p>0.05$; Figure 3G, H, I). Male EE $(2.76 \% \pm 0.375)$ offspring spent a significantly higher percentage of time in the center of the arena compared to SD males $(1.32 \% \pm 0.285)$, though the overall times were quite low.

For the percent of time spent in social interaction, there was a main effect of postnatal experience for male offspring in that postnatal enrichment housing increased social interest (SD: $64.88 \pm 5.36$ vs EE: 81.34 $\pm 3.09 ; \mathrm{F}(1,26)=6.840, \mathrm{p}=0.015, n_{p}{ }^{2}=0.208$; ; Figure 3J, L). For females, the EE prenatal experience increased social engagement level (SD: 49.44 \pm 3.88 vs EE: $75.54 \pm 4.91 ; \mathrm{F}(1,26)=16.140, \mathrm{p}=0.001, n_{p}{ }^{2}=0.383$; Figure 3K, L). Both prenatal $\left(\mathrm{SD}=15.66 \pm 4.37\right.$ and $\left.\mathrm{EE}=7.30 \pm 2.34 ; X^{2}(1)=8.073, \mathrm{p}=0.004\right)$ and postnatal $\left(\mathrm{SD}=17.90 \pm 4.48\right.$ and $\left.\mathrm{EE}=5.06 \pm 1.02 ; X^{2}(1)=4.047, \mathrm{p}=0.044 ;\right)$ enrichment experience decreased the latency for female offspring to approach the novel rat (Figure 3M, $\mathbf{N}, \mathbf{O})$.

\section{Discussion}

In the present study we demonstrate that EE can change the nutritional and microbial profiles of maternal milk in addition to affecting maternal behavior and offspring development. Our results shed light on the multidimensional impact that EE confers on the pre- and neonatal environment and calls attention to the implications of laboratory housing in developmental animal research. While EE was associated with greater bodyweights and sociability in both sexes, the finding that EE dams spent less time on the nest may seem contradictory. However, laboratory rats will spend time away from the nest when given the opportunity, which facilitates more efficient nursing (Ratuski \& Weary, 2021).

A reduced number of nursing posture displays, coupled with a general increase in time away from offspring, may suggest that EE dams are more efficient at nursing while on the nest. SD dams may instead need to shift between nursing postures more frequently in order to maintain an active arched back posture, given the extended periods they spend nursing their young. This may artificially increase the amount of nursing postures they display, alongside the timing of maternal behavior observations (e.g., scoring more frequently in the light or dark phases) which can modulate nursing activity level (Peña \& Champagne, 2013). Notably, $\mathrm{EE}$ and SD dams demonstrated similar amounts of pup licking and grooming despite the differences in time spent on the nest, further supporting the idea that EE dams are more efficient with their care (Welberg et al., 2006; Connors et al., 2015; Strzelewicz et al., 2019; Sale et al., 2004). Higher triglyceride levels in EE milk may compensate for the reduced time on the nest, while SD mothers spend more time nursing to make up for lower milk fat 
content. Alternatively, their milk quality is reduced because they spend more time feeding as they have limited opportunities to distance from their pups and 're-charge'. This is supported by the greater offspring bodyweights of pups that received postnatal EE.

Greater bodyweights are generally associated with better health in wild rats (Barnett, 1958; Barnett \& Dickson, 1984). Heavier adult male wild rats were shown to be more socially integrated within their colony (Barnett \& Dickson, 1984). These results underscore the translational accuracy of EE used in the present study. Increased offspring bodyweights in EE pups may in part be explained by differences in maternal milk quality. Although we did not find differences in the amount of lactose, protein, or IgA in the milk of EE and SD dams, we did find greater triglyceride levels in milk from EE dams at P10. In support of this finding, Chen et al. (2017) found mouse pups that were fostered to dams with greater levels of milk triglycerides weighed significantly more at P12 than pups that were fostered to control mice. Interestingly, while other studies have also demonstrated the role of increased fat consumption on milk triglyceride levels (Ward et al., 2021; Mohammad et al., 2014) our EE and SD dams did not differ in the composition of their diet or bodyweights. Notably, SD dams ate and drank more than EE mothers suggesting that EE positively contributes to milk triglycerides through a different mechanism.

We further validated our finding regarding increased triglycerides in EE milk with microbiome sequencing. Bacteria in maternal milk prime the infant gastrointestinal tract, which can affect its maturation and the future metabolism of nutrients and bacteria (reviewed in depth in Macpherson et al., 2017). Results of microbiome sequencing demonstrated a robust effect of EE on bacterial diversity in milk samples. For example, milk from EE dams had a significantly higher abundance of Christensenellaceae, Peptococcaceae, Lachnospiraceae, Ruminococcaceae, and Erysipelotrichaceae, all of which can directly influence lipid metabolism and body mass index through their manipulation of short-chain fatty acids (Bridgewater et al., 2017; Vacca et al., 2020; Greiner \& Bäckhed, 2011; Waters \& Ley, 2019). Furthermore, milk from EE dams had greater levels of Coriobacteriaceae, which contributes to lipid metabolism (Liu et al., 2018), as well as glucose and steroid metabolism in the gut (Clavel et al., 2014). Although increased Coriobacteriaceae have been found in the ceca of mice exposed to stress (Bangsgaard Bendtsen et al., 2012), it is unlikely our EE dams were significantly more stressed than our SD dams given the lack of significant differences in milk corticosterone and dam body weights. In addition to finding enhanced microbiome diversity in milk from EE dams, microbiome sequencing revealed significantly greater levels of taxa from the Streptococcaceae family in SD dams. Excess expression of this bacterial family in the infant gut has been tied to GI-related issues like dyspepsia and rotavirus infections (Chen et al., 2017; Sohail et al., 2021) which can negatively impact infant growth. Thus, EE may enhance offspring development by altering the microbiome profile of milk that not only promotes the colonization of healthy bacteria, but also discourages exposure to potentially harmful bacteria that can hinder offspring development.

Our observation of increased sociability in EE offspring is in line with several previous studies (Morley-Fletcher et al., 2003; Peña et al., 2006; Schneider et al., 2006; Connors et al., 2014), although these studies utilized EE to rescue social impairments following early life insults. In saline treated control rats, EE was associated with greater time spent in social interaction (Connors et al., 2014), suggesting that EE is not just protective but generally promotes sociability. We expand on these findings by revealing sex- and timespecific effects of EE on behavior in healthy offspring. Research assessing the effects of EE on healthy populations is warranted and positively contributes to the translatability of this housing model (Kentner et al., 2019b; Kentner et al., 2021). Cross-fostering pups between housing conditions after parturition revealed that prenatal enrichment increased sociability in females, while postnatal enrichment increased sociability in males. In addition to differences 
in maternal care, it's plausible that consumption of different milk microbiome profiles may have directly influenced offspring behavior. While the relationship between the gut microbiome and social behavior is well-established (Archie et al., 2015), less is understood about how these bacterial taxa exert their effects on brain development and function once colonized in gut. One study demonstrated the necessity of the HPA-axis and neuronal activation in the hypothalamus and hippocampus in rescuing social behavior in germ free adult male mice (Wu et al., 2021). Since germ-free mice are completely devoid of microbiome, this begs the question of whether bacterial taxa can contribute to behavior on a spectrum, and the results of the present study suggest that they may. However, without an assessment of offspring duodenum taxa, we cannot conclude that the gut microbiome profile of our SD and EE offspring differed.

While the physiological mechanisms that contribute to time- and sex-specific differences in behavior among EE offspring need further elucidation, the present results shed light on maternal care and maternal milk quality as pathways of interest for future studies to explore. Prenatal measures, such as the hormonal milieu during pregnancy, may also be of interest. EE dams gave birth to larger litters, and this may indicate that the benefits of EE can manifest far before parturition. Presumably, the compounding effects of maternal behavior and milk quality contribute to expedited plasticity in the developing brain (Cancedda et al., 2004; Baroncelli et al., 2010).

\section{Conclusions}

The efficaciousness of the "gold standard" housing cages in animal research has recently been called into question (Kentner et al., 2019b; Olsson \& Dahlborn, 2002; Prendergast et al., 2014; Kentner et al., 2021). Results of the present study bolster the argument that EE housing conditions encapsulate a more naturalistic environment than SD, especially with regard to maternal behavior and development (Zhao et al., 2021; Connors et al., 2015; Ratuski \& Weary 2021). Moreover, maternal-fetal interactions are critical to offspring brain development and behavior. Here, we expand on previous studies that have highlighted the beneficial effects of $\mathrm{EE}$ on laboratory rodents, by demonstrating the multifaceted impact of EE on maternal behavior, physiology, and offspring social behavior. These results suggest that the maternal environment contributes to notable, long-term changes in offspring development by more efficient maternal behavior and improved milk quality. Rodent models of breastfeeding are advantageous in teasing apart the mechanisms by which this fascinating substance exerts its influence on brain development and behavior.

\section{Funding and Disclosures}

This project was funded by NIMH under Award Number R15MH114035 (to ACK). The authors wish to thank Mary Erickson and Sahith Kaki for technical support and Dr. Theresa Casey for advice during the early phases of this study. The authors would also like to thank the MCPHS University School of Pharmacy and School of Arts \& Sciences for their continual support. The content is solely the responsibility of the authors and does not necessarily represent the official views of any of the financial supporters.

\section{Author Contributions}

H.D., H.T., A.C.K., ran the experiments; H.D., \& A.C.K. analyzed and interpreted the data, and wrote the manuscript; A.C.K., designed and supervised the study.

\section{Declaration of Competing Interest}

The authors declare that they have no known competing financial interests or personal relationships that could have appeared to influence the work reported in this paper. 


\section{Figure Captions}

Figure 1. Maternal care behaviors are different between environmentally enriched (EE) and standard housed (SD) Sprague-Dawley rat dams. (A) Timeline of experimental procedures. (B) Representative photographs of EE housing and litters. (C) Representative photographs of SD housing and litters. (D) Average number of pups born (male, female, and total pups) per SD and EE housing group $(n=8)$. (E) Total time (seconds) SD and EE housed dams spent exploring postnatal day $(\mathrm{P}) 7$ alien pups from different housing conditions $(\mathrm{n}=9)$. Total time (seconds) that dams spent on the nest across P1-P4 and P10 in the (F) light, (G) dark, and $(\mathrm{H})$ light + dark periods combined. Total time (seconds) that dams spent on the nest collapsed across P1-P4 and P10 in the (I) light, (J) dark, and (K) light + dark periods combined. Stacked bars depict the frequency of pup directed nursing behaviors (arched back nursing, blanked/passive nursing, total nursing) collapsed across P1-P4 and P10 in the (L) light, (M) dark, and (N) light + dark periods. Stacked bars depict the frequency of other types of pup directed behaviors (licking/grooming, pup retrievals, nest building behaviors) collapsed across P1-P4 and P10 in the $(\mathrm{O})$ light, $(\mathrm{P})$ dark, and $(\mathrm{Q})$ light + dark periods. Stacked bars depict the frequency of maternal self-directed behaviors (self-grooming, eating/drinking, tail/arm chases) collapsed across P1-P4 and P10 in the (R) light, (S) dark, and $(\mathrm{T})$ light + dark periods $(\mathrm{n}=8)$. Data are expressed as mean \pm SEM; SD: open circles versus EE: closed circles. ${ }^{*} \mathrm{p}<0.05, * * \mathrm{p}<0.01, * * * \mathrm{p}<0.001$, SD versus EE; ${ }^{\text {aa }} \mathrm{p}<0.01$, main effect of housing; ${ }^{b} \mathrm{p}<0.05$, main effect of postnatal day.

Figure 2. Nutritional profile of milk and microbiome community distribution in environmentally enriched (EE) and standard housed (SD) Sprague-Dawley rat dams. (A) Photograph depictions of maternal milk collection. Maternal milk concentrations $(\mathrm{n}=8)$ of (B) \% creamatocrit, (C) fat $(\mathrm{g} / \mathrm{L})$, (D) energy value $(\mathrm{kcal} / \mathrm{L}),(\mathrm{E})$ protein $(\mathrm{mg} / \mathrm{mL}),(\mathrm{F})$ lactose $(\mathrm{ng} / \mu \mathrm{L}),(\mathrm{G})$ corticosterone $(\mathrm{ng} / \mathrm{mL}),(\mathrm{H}) \mathrm{IgA}(\mathrm{mg} / \mathrm{mL})$, and (I) triglycerides $(\mathrm{mg} / \mathrm{dL})$. (J) Microbiome biomarkers plot $(n=6)$. Taxa identified as significantly more abundant in the milk of the housing group where a bar appears; SD mothers (blue bars) and EE mothers (red bars). Significance was determined by LEfSe analysis, which identified taxa with distributions that were statistically significant $(\mathrm{p}<0.05)$ and where the effect size (LDA score) was greater than 2. (K) Microbial composition of taxonomy in maternal milk at the family level for SD and EE-housed dams $(n=6)$. (L) Alpha diversity along the Shannon Index. (M) Beta diversity using principle coordinate analysis (PCoA). This plot was created using the matrix of pair-wise distance between samples determined by Bray-Curtis dissimilarity using unique amplicon sequencing variants. Each dot represents an individual microbial profile $(n=6)$. Samples that are closer together are more similar, while samples that are dissimilar are plotted further away from one another. Data are expressed as mean \pm SEM; SD: open circles versus EE: closed circles. ${ }^{* *} p<0.01$, SD versus EE; ${ }^{b} p<0.05$, main effect of postnatal day.

Figure 3. Juvenile offspring physiology and behavior following housing in environmentally enriched (EE) or standard housed (SD) laboratory conditions. Data for (left-side) male, (middle) female, and (right-side) male and female Sprague-Dawley rats combined for (A, B, C) P21 body weights (grams). (D, E, F) Total distance traveled $(\mathrm{cm})$, and $(\mathrm{G}, \mathrm{H}, \mathrm{I})$ percent of time spent in the center of an open field. $(\mathrm{J}, \mathrm{K}, \mathrm{L})$ Percent of time spent in social interaction, and (M, N, O) latency (seconds) to approach a novel rat in a social preference test $(n=7-8)$. Data are expressed as mean \pm SEM; SD: open circles versus EE: 
closed circles. ${ }^{\mathrm{aa}} \mathrm{p}<0.01,{ }^{\mathrm{aa}} \mathrm{p}<0.001$, main effect of prenatal experience (SD versus EE); ${ }^{\mathrm{b}} \mathrm{p}<$ $0.05,{ }^{\mathrm{bb}} \mathrm{p}<0.01$, main effect of postnatal experience (SD versus EE).

Supplementary Figure 1. Cladogram of milk biomarkers associated with housing condition (determined by LEfSe). Diameter of the nodes indicates relative abundance of taxa for SD (green) and EE (red) samples. Placement indicates the classification of taxa, where nodes decrease in rank the closer to the center of the diagram.

Supplementary Figure 2. Taxonomy heatmap demonstrating the microbial composition of samples at the species level with the top fifty most abundant species identified. The colored bar at the top indicates housing condition (blue $=\mathrm{SD}$, red $=\mathrm{EE}$ ). Each row represents the abundance for each taxon, with the taxonomy ID shown on the right. Each column represents the abundance for each sample.

\section{References}

Adhisivam, B., Srinivasan, S., Soudarssanane, M. B., Deepak Amalnath, S., \& Nirmal Kumar, A. (2006). Feeding of infants and young children in tsunami affected villages in Pondicherry. Indian Pediatrics, 43(8), 724. PMID: 16951437.

Andreas, N. J., Hyde, M. J., Gale, C., Parkinson, J. R., Jeffries, S., Holmes, E., \& Modi, N. (2014). Effect of maternal body mass index on hormones in breast milk: a systematic review. PLoS One, 9(12), e115043. doi: 10.1371/journal.pone.0115043

Archie, E. A., \& Tung, J. (2015). Social behavior and the microbiome. Current Opinion in Behavioral Sciences, 6, 28-34. https://doi.org/10.1016/j.cobeha.2015.07.008

Bangsgaard Bendtsen, K. M., Krych, L., Sørensen, D. B., Pang, W., Nielsen, D. S., Josefsen, K., ... \& Hansen, A. K. (2012). Gut microbiota composition is correlated to grid floor induced stress and behavior in the BALB/c mouse. PLoS One. 7(10):e46231. doi: 10.1371/journal.pone.0046231.

Baroncelli, L., Braschi, C., Spolidoro, M., Begenisic, T., Sale, A., \& Maffei, L. (2010). Nurturing brain plasticity: impact of environmental enrichment. Cell Death \& Differentiation, 17(7), 1092-1103. https://doi.org/10.1038/cdd.2009.193

Barnett, S. A. (1958). An analysis of social behaviour in wild rats. Proceedings of the Zoological Society of London, 130, (1), 107-152. https://doi.org/10.1111/j.10963642.1958.tb00565.x

Barnett, S. A. (1958). Physiological effects of" social stress" in wild rats. Journal of Psychosomatic Research, 3, 1-11. https://doi.org/10.1016/0022-3999(58)90012-6

Boix-Amorós, A., Collado, M. C., Van’t Land, B., Calvert, A., Le Doare, K., Garssen, J., ... \& Munblit, D. (2019). Reviewing the evidence on breast milk composition and immunological outcomes. Nutrition Reviews, 77(8), 541-556. https://doi.org/10.1093/nutrit/nuz019

Bridgewater, L. C., Zhang, C., Wu, Y., Hu, W., Zhang, Q., Wang, J., ... \& Zhao, L. (2017). 
Gender-based differences in host behavior and gut microbiota composition in response to high fat diet and stress in a mouse model. Scientific Reports, 7(1), 1-12. https://doi.org/10.1038/s41598-017-11069-4

Brion, M. J. A., Lawlor, D. A., Matijasevich, A., Horta, B., Anselmi, L., Araújo, C. L., ... \& Smith, G. D. (2011). What are the causal effects of breastfeeding on IQ, obesity and blood pressure? Evidence from comparing high-income with middle-income cohorts. International Journal of Epidemiology, 40(3), 670-680. doi: 10.1093/ije/dyr020

Buschdorf, J. P., \& Meaney, M. J. (2011). Epigenetics/programming in the HPA axis. Comprehensive Physiology, 6(1), 87-110. doi: 10.1002/cphy.c140027

Callahan B.J., McMurdie P.J., Rosen M.J., Han A.W., Johnson A.J., Holmes S.P., (2016) DADA2: High resolution sample inference from Illumina amplicon data. Nature Methods 13(7):581-3. doi: https://doi.org/10.1038/nmeth.3869

Cancedda, L., Putignano, E., Sale, A., Viegi, A., Berardi, N., \& Maffei, L. (2004). Acceleration of visual system development by environmental enrichment. Journal of Neuroscience, 24(20), 4840-4848. doi: https://doi.org/10.1523/JNEUROSCI.084504.2004

Caporaso, J.G., Kuczynski, J., Stombaugh, J., Bittinger, K., Bushman, F.D., \& Costello, E.K. (2010). QIIME allows analysis of high-throughput community sequencing data. Nature Methods, 7: 335-336. https://doi.org/10.1038/nmeth.f.303

Champagne, F.A. \& Curley, J.P. (2009). Epigenetic mechanisms mediating the long-term effects of maternal care on development. Neuroscience Biobehavioral Reviews, 33(4):593-600. doi: 10.1016/j.neubiorev.2007.10.009.

Chen, Y., Wang, J., Yang, S., Utturkar, S., Crodian, J., Cummings, S., ... \& Casey, T. (2017). Effect of high-fat diet on secreted milk transcriptome in midlactation mice.

Physiological Genomics, 49(12), 747-762. doi:

10.1152/physiolgenomics.00080.2017

Chen, S. Y., Tsai, C. N., Lee, Y. S., Lin, C. Y., Huang, K. Y., Chao, H. C., ... \& Chiu, C. H. (2017). Intestinal microbiome in children with severe and complicated acute viral gastroenteritis. Scientific Reports, 7(1), 1-7. doi: 10.1038/srep46130

Chiba, T., Maeda, T., Fujita, Y., Takeda, R., Kikuchi, A., \& Kudo, K. (2019) Stress-induced suppression of milk protein is involved in a noradrenergic mechanism in the mammary gland. Endocrinology, 160(9), 2074-2084.

https://doi.org/10.1210/en.2019-00300

Clavel, T., Desmarchelier, C., Haller, D., Gérard, P., Rohn, S., Lepage, P., \& Daniel, H. (2014). Intestinal microbiota in metabolic diseases: from bacterial community structure and functions to species of pathophysiological relevance. Gut Microbes, 5(4), 544-551. doi: 10.4161/gmic.29331

Connors, E. J., Migliore, M. M., Pillsbury, S. L., Shaik, A. N., \& Kentner, A. C. (2015). 
Environmental enrichment models a naturalistic form of maternal separation and shapes the anxiety response patterns of offspring. Psychoneuroendocrinology, 52, 153-167. doi: 10.1016/j.psyneuen.2014.10.021

Connors, E. J., Shaik, A. N., Migliore, M. M., \& Kentner, A. C. (2014). Environmental enrichment mitigates the sex-specific effects of gestational inflammation on social engagement and the hypothalamic pituitary adrenal axis-feedback system. Brain, Behavior, and Immunity, 42, 178-190. https://doi.org/10.1016/j.bbi.2014.06.020

Cramer, C. P., Thiels, E., \& Alberts, J. R. (1990). Weaning in rats: I. Maternal behavior. Developmental Psychobiology: The Journal of the International Society for Developmental Psychobiology, 23(6), 479-493. https://doi.org/10.1002/dev.420230604

Crawley, J.N., 2007. Social behavior tests for mice. In: Crawley, J.N. (Ed.), What's Wrong with My Mouse? Strategies for Rodent Behavior Phenotyping. Society for Neuroscience, San Diego, pp. 63-70. doi:10.1002/9780470119051

DeYoung, S.E., Chase, J., Branco, M.P. \& Park, B. (2018). The effect of mass evacuation on infant feeding: The case of the 2016 Fort McMurray wildfire. Maternal Child Health Journal, 22, 1826-1833. https://doi.org/10.1007/s10995-018-2585-z

Di Benedetto, M. G., Bottanelli, C., Cattaneo, A., Pariante, C. M., \& Borsini, A. (2020). Nutritional and immunological factors in breast milk: A role in the intergenerational transmission from maternal psychopathology to child development. Brain, Behavior, and Immunity, 85, 57-68. doi: 10.1016/j.bbi.2019.05.032

Drugs and Lactation Database (LactMed) [Internet]. Bethesda (MD): National Library of Medicine (US); 2006-. Isoflurane. [Updated 2020 Nov 16]. Bookshelf URL: https://www.ncbi.nlm.nih.gov/books/

Duque-Wilckens, N., Torres, L.Y., Yokoyama, S., Minie, V.A., Tran, A.M., Petkova, S.P., Hao, R., Ramos-Maciel, S., Rios, R.A., Jackson, K., Flores-Ramirez, F.J., GarciaCarachure, I., Pesavento, P.A., Iniguez, S.D., Grinevich, V., \& Trainor, B.C. (2020). Extrahypothalamicoxytocin neurons drive stress-induced social vigilance and avoidance. PNAS U S A. 5:202011890. doi: 10.1073/pnas.2011890117.

Frank, N. M., Lynch, K. F., Uusitalo, U., Yang, J., Lönnrot, M., Virtanen, S. M., ... \& Norris, J. M. (2019). The relationship between breastfeeding and reported respiratory and gastrointestinal infection rates in young children. BMC Pediatrics, 19(1), 1-12. https://doi.org/10.1186/s12887-019-1693-2

Gaskill, B. N., \& Pritchett-Corning, K. R. (2015). The effect of cage space on behavior and reproduction in $\mathrm{Crl}$ : $\mathrm{CD} 1$ (Icr) and $\mathrm{C} 57 \mathrm{BL} / 6 \mathrm{NCrl}$ laboratory mice. PLoS One, 10(5), e0127875. doi: 10.1371/journal.pone.0127875

Grace, T., Oddy, W., Bulsara, M., \& Hands, B. (2017). Breastfeeding and motor development: A longitudinal cohort study. Human Movement Science, 51, 9-16.

Gray, K. D., Hannon, E. A., Erickson, E., Stewart, A. B., Wood, C. T., Fisher, K., ... \& 
Tanaka, D. (2021). Influence of early lactation assistance on inpatient exclusive breastfeeding rates. Journal of Human Lactation, 37(3), 556-565. doi: $10.1177 / 0890334420957967$.

Greiner, T., \& Bäckhed, F. (2011). Effects of the gut microbiota on obesity and glucose homeostasis. Trends in Endocrinology \& Metabolism, 22(4), 117-123. doi: 10.1016/j.tem.2011.01.002.

Groer, M., Davis, M., \& Steele, K. (2004). Associations between human milk SIgA and maternal immune, infectious, endocrine, and stress variables. Journal of Human Lactation, 20(2), 153-158. doi: 10.1177/0890334404264104

Grota, L.J. \& Ader, R. Continuous recording of maternal behaviour in Rattus norvegicus. (1969) Animal Behavior. 17(4). https://doi.org/10.1016/S0003-3472(69)80019-9

Haldar, J. and V. Bade. 1981. Involvement of opioid peptides in the inhibition of oxytocin release by heat stress in lactating mice. Experimental Biology and Medicine (Maywood). 168:10-14. DOI: 10.3181/00379727-168-41227

Hanson, L. A. (1998). Breastfeeding provides passive and likely long-lasting active immunity. Annals of Allergy, Asthma \& Immunology, 81(6), 523-537. https://doi.org/10.1016/S1081-1206(10)62704-4

Horwood, L. J., \& Fergusson, D. M. (1998). Breastfeeding and later cognitive and academic outcomes. Pediatrics, 101(1), e9-e9. doi: 10.1542/peds.101.1.e9.

Hughes, C., Harlan, R., \& Plaut, S. (1978). Maternal behavior of wild and domestic Rattus norvegicus recorded continuously in dual-chambered cages. Developmental Psychobiology, 11(4):329-34. https://doi.org/10.1002/dev.420110406

Huff K, Suárez-Trujillo A, Kuang S, Plaut K, Casey T. (2020). One-to-one relationships between milk miRNA content and protein abundance in neonate duodenum support the potential for milk miRNAs regulating neonate development. Functional and Integrative Genomics.20(5):645-656. doi: 10.1007/s10142-020-00743-y

Isaacs, E. B., Fischl, B. R., Quinn, B. T., Chong, W. K., Gadian, D. G., \& Lucas, A. (2010). Impact of breast milk on intelligence quotient, brain size, and white matter development. Pediatric Research, 67(4), 357-362. https://doi.org/10.1203/PDR.0b013e3181d026da

Jankowsky, J. L., Melnikova, T., Fadale, D. J., Xu, G. M., Slunt, H. H., Gonzales, V., ... \& Savonenko, A. V. (2005). Environmental enrichment mitigates cognitive deficits in a mouse model of Alzheimer's disease. Journal of Neuroscience, 25(21), 5217-5224. doi: 10.1523/JNEUROSCI.5080-04.2005

Kanazawa, S. (2015). Breastfeeding is positively associated with child intelligence even net of parental IQ. Developmental Psychology, 51(12), 1683. doi: 10.1037/dev0000060

Keen, C. L., Lönnerdal, B. O., Clegg, M., \& Hurley, L. S. (1981). Developmental changes in 
composition of rat milk: trace elements, minerals, protein, carbohydrate and fat. The Journal of Nutrition, 111(2), 226-236. doi: 10.1093/jn/111.2.226

Kentner, A. C., Lambert, K. G., Hannan, A. J., \& Donaldson, S. T. (2019a). Environmental enrichment: Enhancing neural plasticity, resilience, and repair. Frontiers in Behavioral Neuroscience, 13, 75. https://doi.org/10.3389/fnbeh.2019.00075

Kentner, A.C., Cryan, J.F., Brummelte, S. (2019b). Resilience priming: translational models for understanding resiliency and adaptation to early life adversity. Developmental Psychobiology, 61, 350-375. https://doi.org/10.1002/dev.21775

Kentner, A. C., Khoury, A., Queiroz, E. L., \& MacRae, M. (2016). Environmental enrichment rescues the effects of early life inflammation on markers of synaptic transmission and plasticity. Brain, Behavior, and Immunity, 57, 151-160. doi: 10.1016/j.bbi.2016.03.013.

Kentner, A.C., Speno, A.V., Doucette, J., Roderick, R.C. (2021). The contribution of environmental enrichment to phenotypic variation in mice and rats. eNeuro, 8 , ENERUO.0539-20.2021, doi: 10.1523/ENEURO.0539-20.2021

Kotloski, R. J. \& Sutula, T. P. (2015). Environmental enrichment: evidence for an unexpected therapeutic influence. Experimental Neurology, 264, 121-126. doi: 10.1016/j.expneurol.2014.11.012

Lauritzen, L., Brambilla, P., Mazzocchi, A., Harsløf, L., Ciappolino, V., \& Agostoni, C. (2016). DHA effects in brain development and function. Nutrients, 8(1), 6.

Lee, J.J. \& Rubin, A.P. (2993). Breast feeding and anesthesia. Anesthesia, 48, 616-625, doi: 10.1111/j.1365-2044.1993.tb07130.x.

Lessen, R., \& Kavanagh, K. (2015). Position of the academy of nutrition and dietetics: promoting and supporting breastfeeding. Journal of the Academy of Nutrition and Dietetics, 115(3), 444-449. https://doi.org/10.1016/j.jand.2014.12.014

Liu, H., Zhang, H., Wang, X., Yu, X., Hu, C., \& Zhang, X. (2018). The family Coriobacteriaceae is a potential contributor to the beneficial effects of Roux-en-Y gastric bypass on type 2 diabetes. Surgery for Obesity and Related Diseases, 14(5), 584-593. doi: 10.1016/j.soard.2018.01.012

Luby, J. L., Belden, A. C., Whalen, D., Harms, M. P., \& Barch, D. M. (2016). Breastfeeding and childhood IQ: The mediating role of gray matter volume. Journal of the American Academy of Child \& Adolescent Psychiatry, 55(5), 367-375. doi: 10.1016/j.jaac.2016.02.009

Lucas, A., Gibbs, J.A., Lyster, R.L., Baum, J.D. (1978). Creamatocrit: simple clinical technique for estimating fat concentration and energy value of human milk. British Medical Journal, 1 (6119):1018-1020, doi: 10.1136/bmj.1.6119.1018.

Macpherson, A. J., de Agüero, M. G., \& Ganal-Vonarburg, S. C. (2017). How nutrition and 
the maternal microbiota shape the neonatal immune system. Nature Reviews Immunology, 17(8), 508-517. https://doi.org/10.1038/nri.2017.58

Mann, P. E., \& Gervais, K. J. (2011). Environmental enrichment delays pup $\square$ induced maternal behavior in rats. Developmental Psychobiology, 53(4), 371-382. doi: $10.1002 / \mathrm{dev} .20526$

Meaney, M. J., \& Szyf, M. (2005). Maternal care as a model for experience-dependent chromatin plasticity?. Trends in Neurosciences, 28(9), 456-463. doi: 10.1016/j.tins.2005.07.006.

Meaney, M. J. (2010). Epigenetics and the biological definition of gene $\times$ environment interactions. Child Development, 81(1), 41-79. doi: 10.1111/j.14678624.2009.01381.x.

Meeh, K. L., Rickel, C. T., Sansano, A. J., \& Shirangi, T. R. (2021). The development of sex differences in the nervous system and behavior of flies, worms, and rodents. Developmental Biology. https://doi.org/10.1016/j.ydbio.2021.01.010

Miles, J. \& Shevlin, M., 2001. Applying regression and correlation: A guide for students and researchers. Sage.

Mohammad, M. A., Sunehag, A. L., \& Haymond, M. W. (2014). De novo synthesis of milk triglycerides in humans. American Journal of Physiology-Endocrinology and Metabolism, 306(7), E838-E847. doi: 10.1152/ajpendo.00605.2013

Moirasgenti, M., Doulougeri, K., Panagopoulou, E., \& Theodoridis, T. (2019). Psychological stress reduces the immunological benefits of breast milk. Stress and Health, 35(5), 681-685. doi: 10.1002/smi.2903.

Murgatroyd, C., Taliefar, M., Bradburn, S....Nephew, B. (2015). Social stress during lactation, depressed maternal care, and neuropeptidergic gene expression. Behavioural Pharmacology, 26, 642-653. doi: 10.1097/FBP.0000000000000147.

Olsson, I. A. S., \& Dahlborn, K. (2002). Improving housing conditions for laboratory mice: a review of 'environmental enrichment'. Laboratory Animals, 36(3), 243-270.

Ordoñes Sanchez, E., Bavley, C.C., Deutschmann, A.U., Carpenter, R., Peterson, D.R., Karbalaei, R....Bangasser, D.A. (2021). Early life adversity promotes resilience to opioid addiction-related phenotypes in male rats and sex-specific transcriptional changes. PNAS USA, 118, e2020173118. doi: 10.1073/pnas.2020173118.

Pannaraj, P. S., Li, F., Cerini, C., Bender, J. M., Yang, S., Rollie, A., ... \& Aldrovandi, G. M. (2017). Association between breast milk bacterial communities and establishment and development of the infant gut microbiome. JAMA Pediatrics, 171(7), 647-654. doi:10.1001/jamapediatrics.2017.0378

Par Pharmaceutical, Inc, Pitocin [Label], Par Pharmaceutical, Chestnut Ridge, NY, 2020, https://dailymed.nlm.nih.gov/dailymed/fda/fdaDrugXsl.cfm?setid=6d4b2c25-2e5d49b5-93bc-2ae8a20916d1\&type=display 
Paul, H.A., Hallam, M.C., Reimer, R.A. (2015). Milk collection in the rat using capillary tubes and estimation of milk fat content by creamatocrit. Journal of Visualized Experiments, 106, 1-6, doi: 10.3791/53476.

Peña, C. J., Champagne, F. A. (2013). Implications of temporal variation in maternal care for the prediction of neurobiological and behavioral outcomes in offspring. Behavioral Neuroscience, 127 (1), 33-46. doi: 10.1037/a0031219

Prendergast, B. J., Onishi, K. G., \& Zucker, I. (2014). Female mice liberated for inclusion in neuroscience and biomedical research. Neuroscience \& Biobehavioral Reviews, 40, 1-5. https://doi.org/10.1016/j.neubiorev.2014.01.001

Raine, A., Mellingen, K., Liu, J., Venables, P., \& Mednick, S. A. (2003). Effects of environmental enrichment at ages 3-5 years on schizotypal personality and antisocial behavior at ages 17 and 23 years. American Journal of Psychiatry, 160(9), 16271635. doi: 10.1176/appi.ajp.160.9.1627

Ratuski A.S. \& Weary, D.M. (2021). A break from the pups: The effects of loft access on the welfare of lactating laboratory rats. PLoS One. 16(6):e0253020. doi:

10.1371/journal.pone.0253020.

Ribo, S., Sánchez-Infantes, D., Martinez-Guino, L., García-Mantrana, I., Ramon-Krauel, M., Tondo, M., ... \& Lerin, C. (2021). Increasing breast milk betaine modulates Akkermansia abundance in mammalian neonates and improves long-term metabolic health. Science Translational Medicine, 13(587). doi: 10.1126/scitranslmed.abb0322

Romero Ramírez, D. S., Lara Pérez, M. M., Carretero Pérez, M., Suárez Hernández, M. I., Martín Pulido, S., Pera Villacampa, L., ... \& García Bello, M. Á. (2021). SARS-CoV2 antibodies in breast milk after vaccination. Pediatrics, $148(5)$. doi: 10.1542/peds.2021-052286.

Sadauskaitė $\square$ Kuehne, V., Ludvigsson, J., Padaiga, Ž., Jašinskienè, E., \& Samuelsson, U. (2004). Longer breastfeeding is an independent protective factor against development of type 1 diabetes mellitus in childhood. Diabetes/Metabolism Research and Reviews, 20(2), 150-157. doi: 10.1002/dmrr.425

Sale, A., Putignano, E., Cancedda, L., Landi, S., Cirulli, F., Berardi, N., Maffei, L. (2004). Enriched environment and acceleration of visual system development. Neuropharmacology, 47, 649-660. doi: 10.1016/j.neuropharm.2004.07.008

Sauerwald, T. U., Demmelmair, H., \& Koletzko, B. (2001). Polyunsaturated fatty acid supply with human milk. Lipids, 36(9), 991-996. doi: 10.1007/s11745-001-0810-9

Schellekens, H., Torres-Fuentes, C., van de Wouw, M., Long-Smith, C. M., Mitchell, A., Strain, C., ... \& Cryan, J. F. (2021). Bifidobacterium longum counters the effects of obesity: Partial successful translation from rodent to human. EBioMedicine, 63, 103176. doi: https://doi.org/10.1016/j.ebiom.2020.103176

Segata, N., Izard, J., Waldron, L., Gevers, D., Miropolsky, L., Garrett, W.S., \& Huttenhower, 
C. (2011) Metagenomic biomarker discovery and explanation. Genome Biology, 12: R60. doi: 10.1186/gb-2011-12-6-r60

Sheard, N. F., \& Walker, W. A. (1988). The role of breast milk in the development of the gastrointestinal tract. Nutrition Reviews (USA). doi: 10.1111/j.17534887.1988.tb05343.x.

Smit, E. N., Oelen, E. A., Seerat, E., Muskiet, F. A., \& Boersma, E. R. (2000). Breast milk docosahexaenoic acid (DHA) correlates with DHA status of malnourished infants. Archives of Disease in Childhood, 82(6), 493-494. http://dx.doi.org/10.1136/adc.82.6.493

Sohail, M. U., Al Khatib, H. A., Al Thani, A. A., Al Ansari, K., Yassine, H. M., \& AlAsmakh, M. (2021). Microbiome profiling of rotavirus infected children suffering from acute gastroenteritis. Gut Pathogens, 13(1), 1-9. https://doi.org/10.1186/s13099021-00411-X

Spring, P. C. M., Amancio, O. M. S., Nobriga, F., Araujo, G., Koppel, S. M., \& Dodge, J. A. (1985). Fat and energy content of breast milk of malnourished and well nourished women, Brazil 1982. Annals of Tropical Paediatrics, 5(2), 83-87. doi: 10.1080/02724936.1985.11748368.

Strzelewicz, A.R., Vecciarelli, H.A., Rondón-Ortiz, Raneri, A., Hill., M.J., Kentner, A.C. (2021). Interactive effects of compounding multidimensional stressors on maternal and male and female rat offspring outcomes. Hormones and Behavior, 134, 105013, doi: 10.1016/j.yhbeh.2021.105013.

Strzelewicz, A.R, Ordoñes Sanchez, E., Rondón-Ortiz, A.N., Raneri, A., Famularo, S.T., Bangasser, D.A., Kentner, A.C. (2019). Access to a high resource environment protects against accelerated maturation following early life stress: a translational animal model of high, medium, and low security settings. Hormones and Behavior, 111, 46-59. doi: 10.1016/j.yhbeh.2019.01.003.

Tooley, U.A., Bassett, D.S. \& Mackey, A.P. (2021). Environmental influences on the pace of brain development. Nature Review Neuroscience. 22(6):372-384. doi: 10.1038/s41583-021- 00457-5

Vacca, M., Celano, G., Calabrese, F. M., Portincasa, P., Gobbetti, M., \& De Angelis, M. (2020). The controversial role of human gut lachnospiraceae. Microorganisms, 8(4), 573. doi: 10.3390/microorganisms 8040573

Ward, E., Yang, N., Muhlhausler, B. S., Leghi, G. E., Netting, M. J., Elmes, M. J., \& Langley $\square$ Evans, S. C. (2021). Acute changes to breast milk composition following consumption of high $\square$ fat and high $\square$ sugar meals. Maternal \& Child Nutrition, 17(3), e13168. doi: 10.1111/mcn.13168.

Waters, J. L., \& Ley, R. E. (2019). The human gut bacteria Christensenellaceae are widespread, heritable, and associated with health. BMC Biology, 17(1), 1-11. https://doi.org/10.1186/s12915-019-0699-4 
Welberg, L., Thrivikraman, K. V., \& Plotsky, P. M. (2006). Combined pre-and postnatal environmental enrichment programs the HPA axis differentially in male and female rats. Psychoneuroendocrinology, 31(5), 553-564. doi: 10.1016/j.psyneuen.2005

Williams, A.V., Duque-Wilckens, N., Ramos-Maciel, S., Campi, K.L., Bhela, S.K., Xu, C.K., Jackson, K., Chini, B., Pesavento, P.A., Trainor, B.C., 2020. Social approach and social vigilance are differentially regulated by oxytocin receptors in the nucleus accumbens. Neuropsychopharmacology, 45, 1423-1430. https://doi.org/10.1038/ s41386-020-0657-4.

Wu, W.L., Adame, M.D., Liou, C.W., Barlow, J.T., Lai, T.T., Sharon, G., Schretter, C.E., Needham, B.D., Wang, M.I., Tang, W., Ousey, J., Lin, Y.Y., Yao, T.H., Abdel-Haq, R., Beadle, K., Gradinaru, V., Ismagilov, R.F., \& Mazmanian, S.K. (2021).

Microbiota regulate social behaviour via stress response neurons in the brain. Nature. 595(7867):409-414. doi: 10.1038/s41586-021-03669-y

Zhao, X., Mohammed, R., Tran, H., Erickson, M., \& Kentner, A.C. (2021). Poly (I:C)induced maternal immune activation modifies ventral hippocampal regulation of stress reactivity: prevention by environmental enrichment. Brain Behavior Immunity, 95, 203-215. doi: 10.1016/j.bbi.2021.03.018

Ziomkiewicz, A., Babiszewska, M., Apanasewicz, A., Piosek, M., Wychowaniec, P., Cierniak, A., ... \& Wichary, S. (2021). Psychosocial stress and cortisol stress reactivity predict breast milk composition. Scientific Reports, 11(1), 1-14. https://doi.org/10.1038/s41598-021-90980-3

Zuena, A. R., Zinni, M., Giuli, C., Cinque, C., Alemà, G. S., Giuliani, A., ... \& Cozzolino, R. (2016). Maternal exposure to environmental enrichment before and during gestation influences behaviour of rat offspring in a sex-specific manner. Physiology \& Behavior, 163, 274-287. doi: 10.1016/j.physbeh.2016.05.010. 


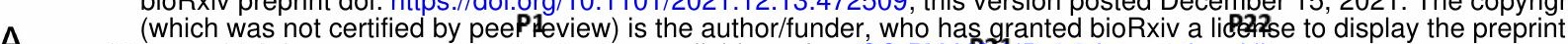

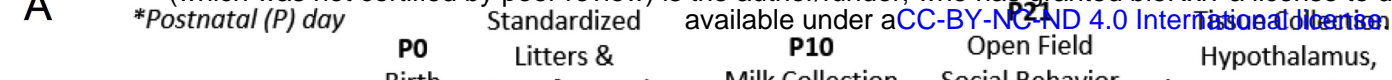

$\begin{aligned} & \text { Breeding Birth } \\ & \text { Cross-fostered }\end{aligned}$ Milk Collection Social Behavior Hippocampus, Amygdala
Mimals arrived
mediately assigned
Standard" (SD) or
"Environmental

B
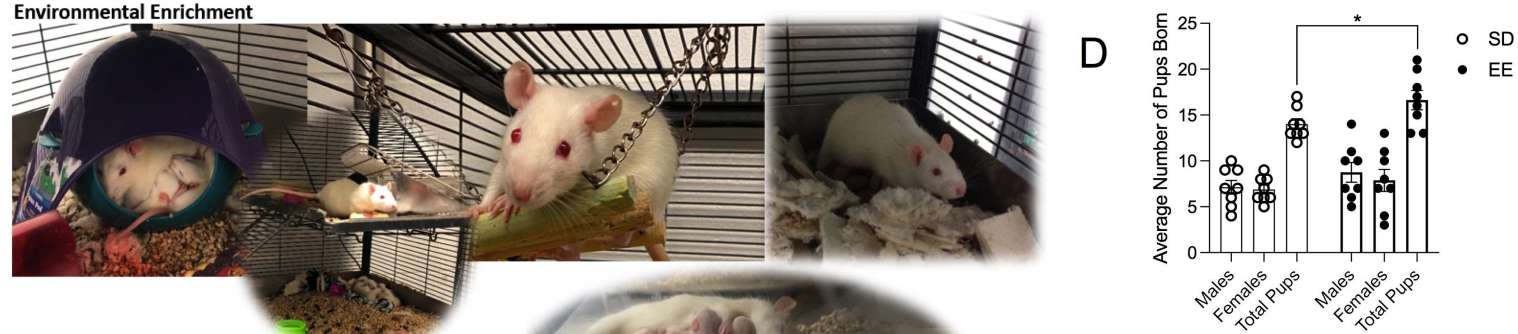

\section{Standard Laboratory Housing}

C
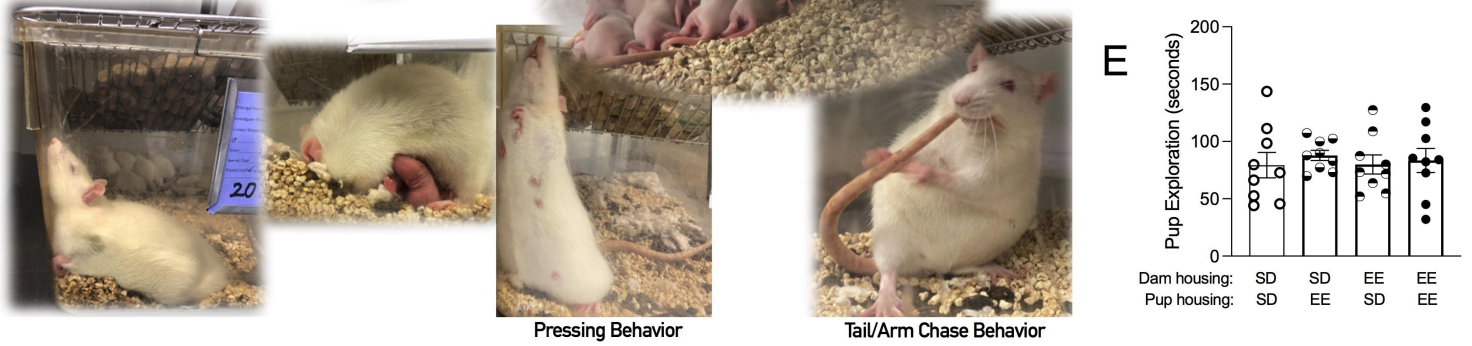

$\mathrm{F}$
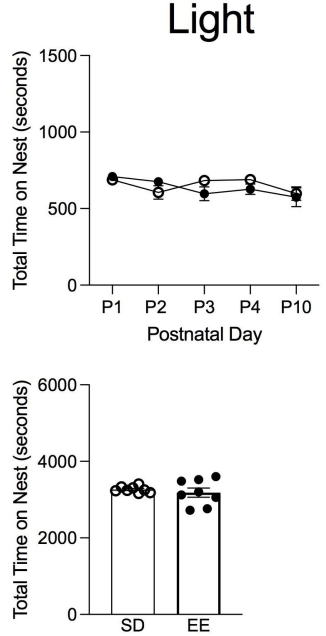

L

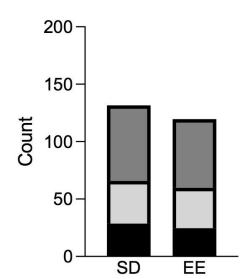

O

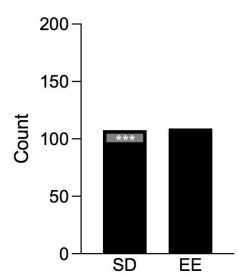

R

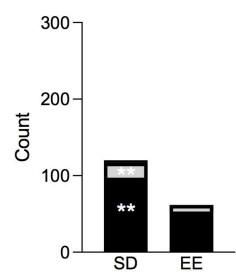

Dark

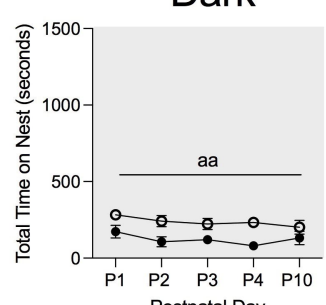

$\mathrm{J}$

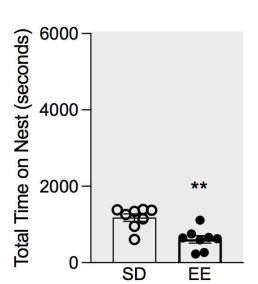

M

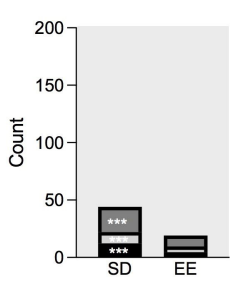

P

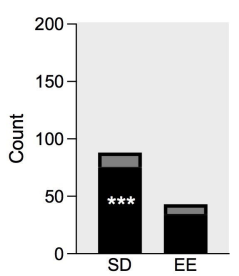

$S$

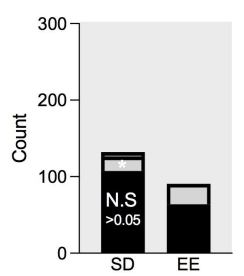

$\mathrm{H}$

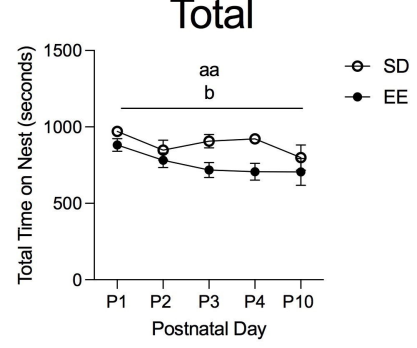

K

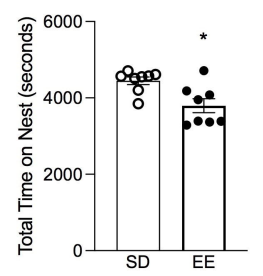

$\mathrm{N}$

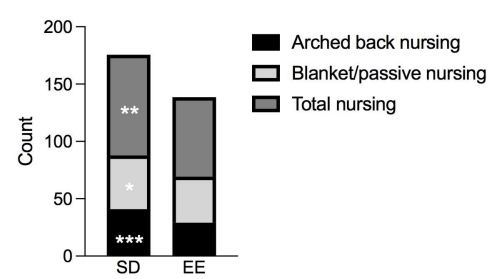

Q
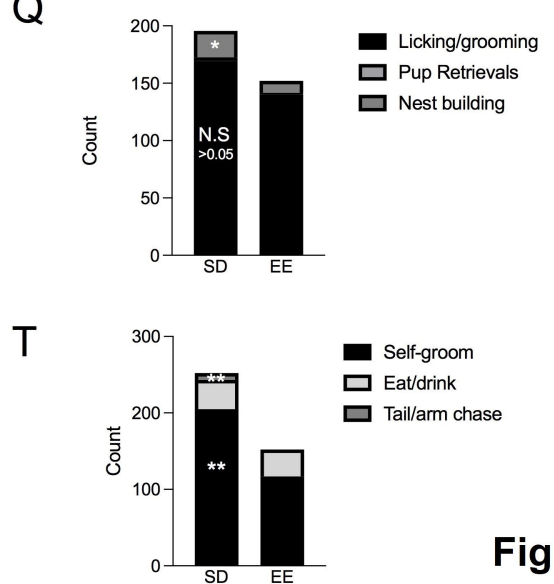

Figure 1 
bioRxiv preprint doi: https://doi.oR/10.1101/2021.12.13.472509; this version posted December 15, 202 $\mathrm{P}$ The copyright holder for this preprint

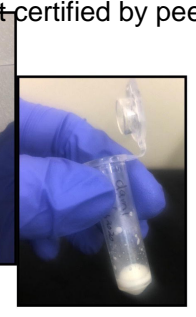

E

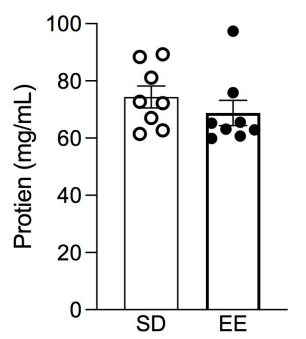

$\mathrm{F}$

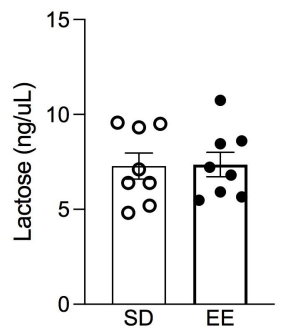

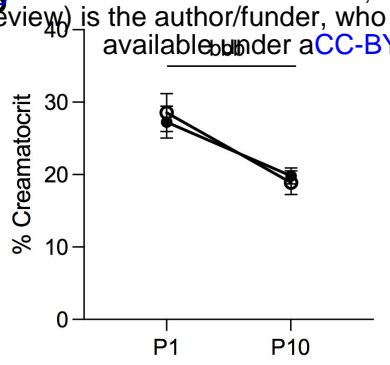

G

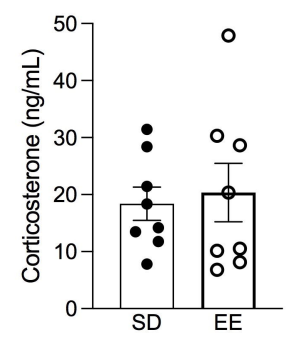

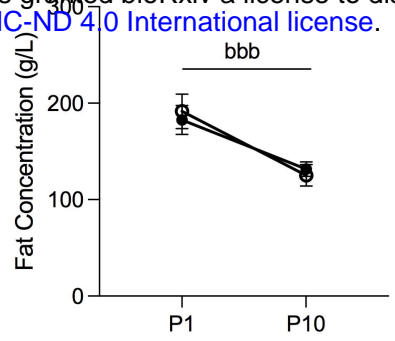

$\mathrm{H}$

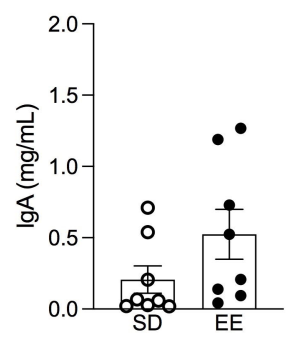

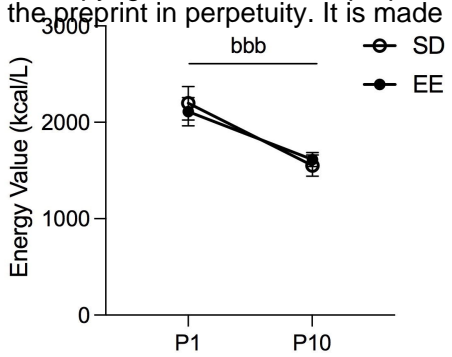

I

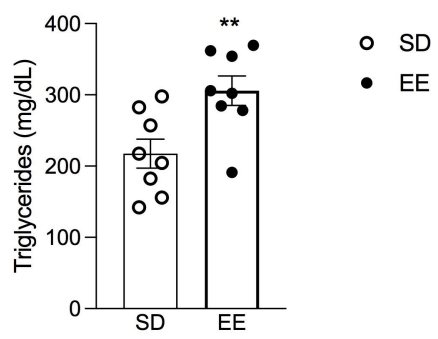

K SD EE

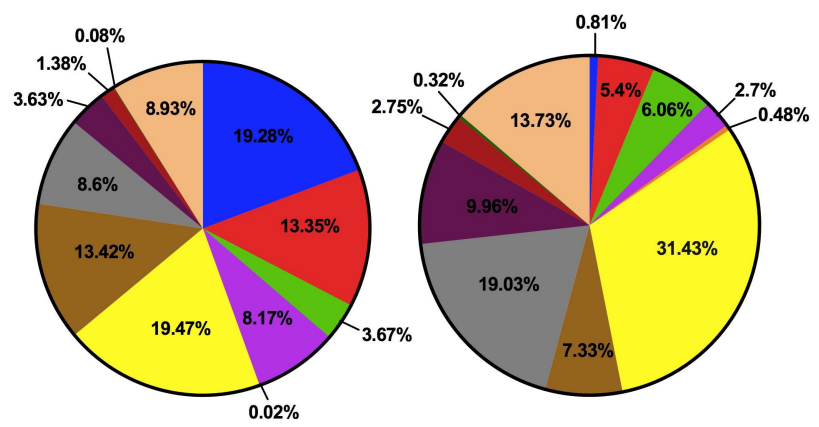

SD EE

\section{Biomarkers Ordered By Effect Size (LDA Score)}

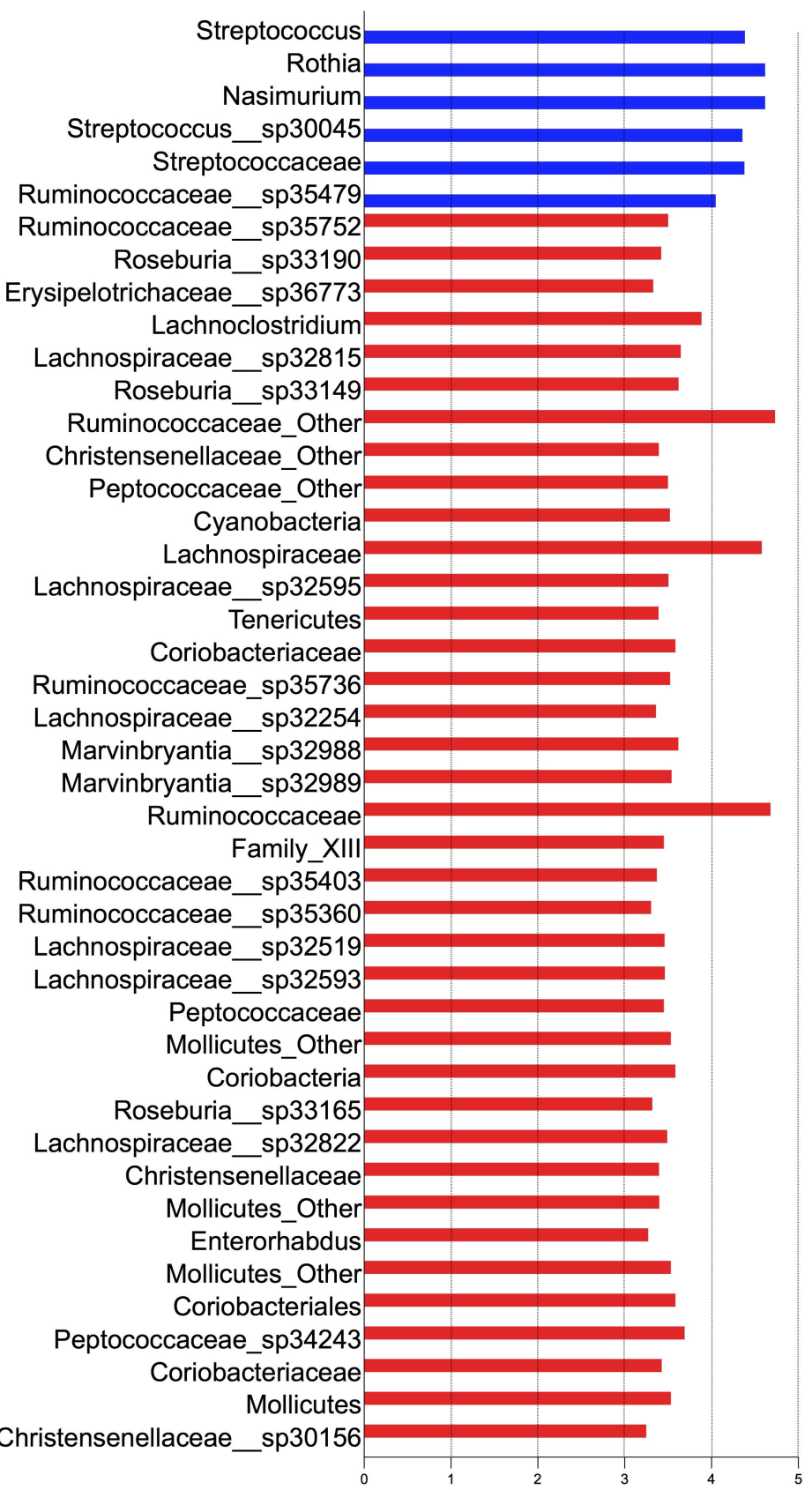


A

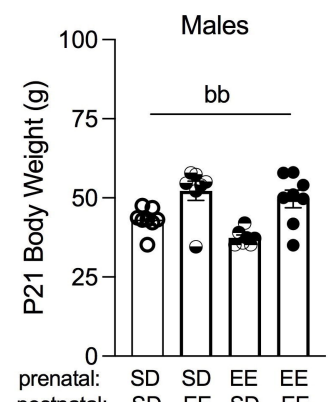
postnatal: SD EE SD EE

D

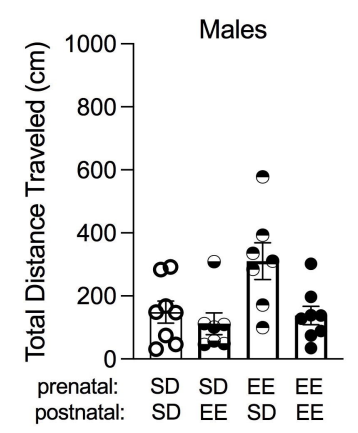

G

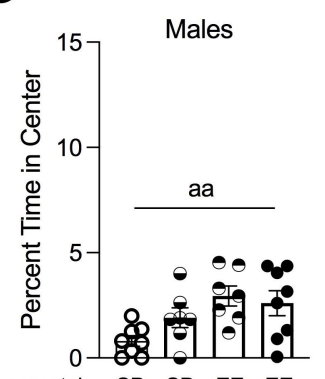

prenatal: SD SD EE EE postnatal: $\mathrm{SD}$ EE SD EE

$\mathrm{J}$

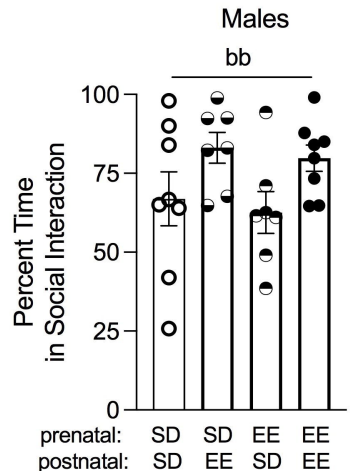

M

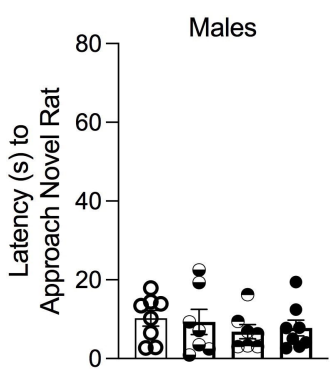

prenatal: SD SD EE EE postnatal: SD EE SD EE
B

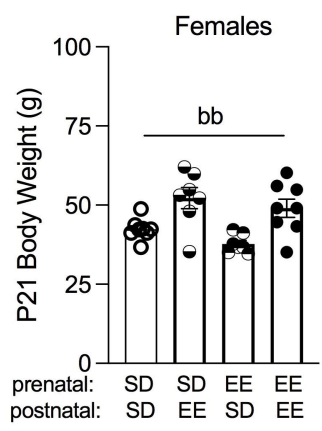

E

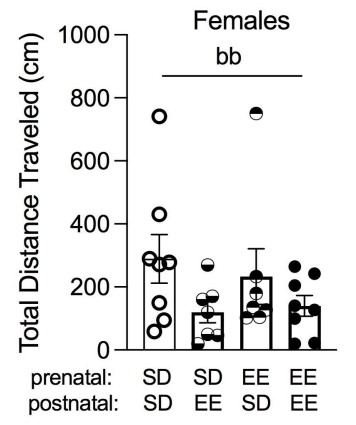

$\mathrm{H}$

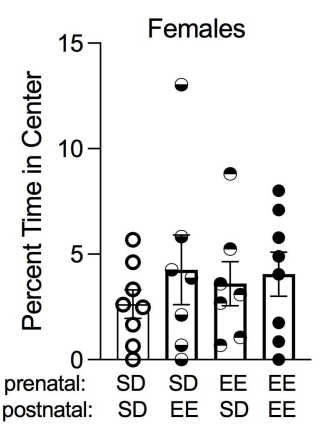

$\mathrm{K}$

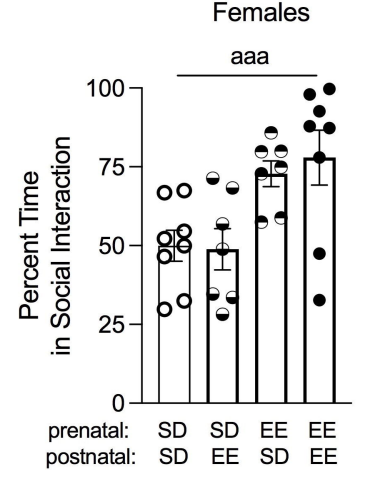

N

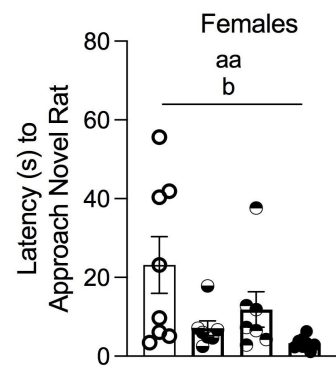

prenatal: SD SD EE EE postnatal: SD EE SD EE

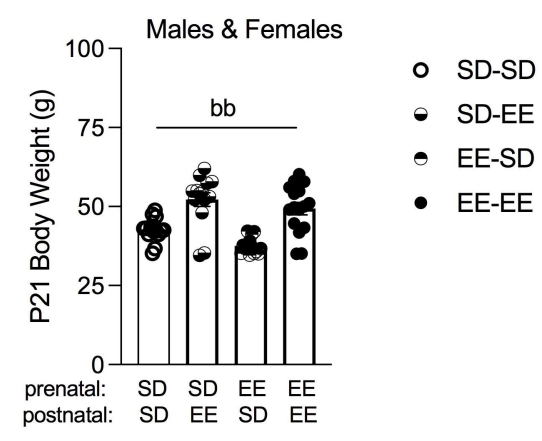

$\mathrm{F}$
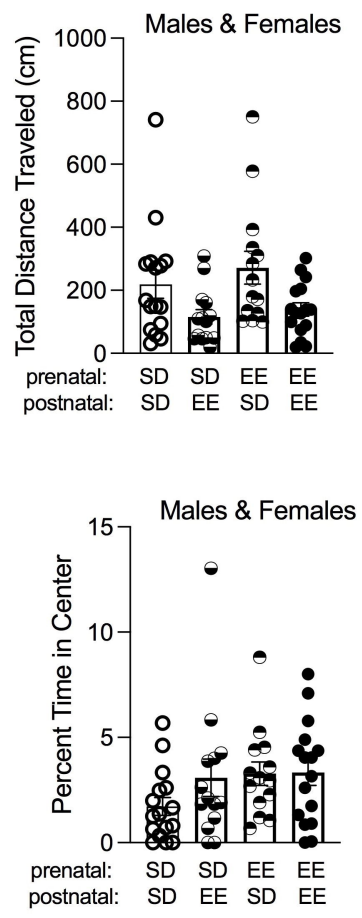

Males and Females

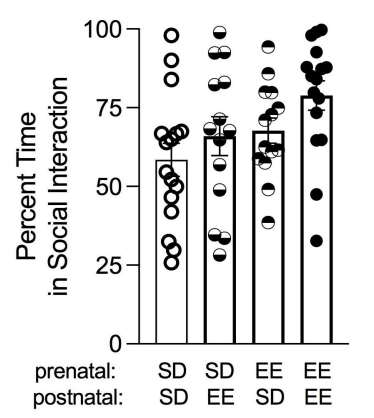

O

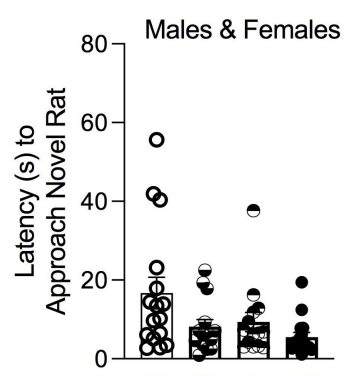

prenatal: SD SD EE EE postnatal: SD EE SD EE 